\title{
A novel efficient and precise technique for removing acetabular osteophytes in patients undergoing total hip arthroplasty: the SH-9Hospital acetabular edge file
}

\author{
Yongyun Chang ${ }^{1,2 \#}$, Keyu Kong ${ }^{1 \#}$, Yiming Zeng ${ }^{1 \#}$, Degang Yu ${ }^{1}$, Jingwei Zhang ${ }^{1}$, Mengning Yan ${ }^{1}$, \\ Yuanqing $\mathrm{Mao}^{1}$, Huiwu Li ${ }^{1}$, Zanjing Zhai ${ }^{1}$ \\ ${ }^{1}$ Shanghai Key Laboratory of Orthopaedic Implants, Department of Orthopaedic Surgery, Shanghai Ninth People's Hospital, Shanghai Jiao Tong \\ University School of Medicine, Shanghai, China; ${ }^{2}$ Department of Orthopaedic Surgery, Renji Hospital, School of Medicine, Shanghai Jiao Tong \\ University, Shanghai, China \\ Contributions: (I) Conception and design: H Li, Z Zhai; (II) Administrative support: Y Mao; (III) Provision of study materials or patients: M Yan; \\ (IV) Collection and assembly of data: D Yu, J Zhang; (V) Data analysis and interpretation: Y Chang, K Kong, Y Zeng; (VI) Manuscript writing: All \\ authors; (VII) Final approval of manuscript: All authors. \\ "These authors contributed equally to this work. \\ Correspondence to: Zanjing Zhai; Huiwu Li. Shanghai Key Laboratory of Orthopaedic Implants, Department of Orthopaedic Surgery, Shanghai Ninth \\ People's Hospital, Shanghai Jiao Tong University School of Medicine, Shanghai, China. Email: zanjing_zhai@163.com; huiwu1223@163.com.
}

\begin{abstract}
Background: Total hip arthroplasty (THA) is frequently performed in patients with end-stage hip disease. Periacetabular osteophytes are common during THA; however, these osteophytes should be removed intraoperatively to avoid potential impingement between osteophytes and femoral prostheses and decrease dislocation risk. There are no current standard procedures or surgical technique criteria to remove these osteophytes. Osteophytes around the acetabulum are usually removed with an osteotome, yet this presents certain disadvantages. Hence, this study aimed to introduce a novel and more efficient technique than the aforementioned one, the SH-9Hospital acetabular edge file.
\end{abstract}

Methods: Fifty-four patients (54 hips) who underwent primary THA using osteotome and the SH-9Hospital acetabular edge file to remove periacetabular osteophytes intraoperatively were retrospectively studied. Clinical and radiographic data were obtained for all patients intra- and postoperatively.

Results: The mean osteophyte removal time was $274.6 \pm 102.7 \mathrm{~s}$ and $51.3 \pm 21.1 \mathrm{~s}$ in the osteotome and SH-9Hospital acetabular edge file groups, respectively. Intraoperative images and postoperative radiographs showed that acetabular osteophytes were removed thoroughly and precisely by the acetabular edge file and that there was no iatrogenic injury and prostheses malposition in both groups.

Conclusions: The SH-9Hospital acetabular edge file was a novel, efficient, highly precise, and repeatable method for removing periacetabular osteophytes in patients undergoing total hip arthroplasty.

Keywords: Total hip arthroplasty (THA); osteophytes; acetabulum; osteotome; SH-9Hospital acetabular edge file

Submitted May 10, 2021. Accepted for publication Jul 20, 2021.

doi: $10.21037 / \mathrm{atm}-21-2400$

View this article at: https://dx.doi.org/10.21037/atm-21-2400

\section{Introduction}

Total hip arthroplasty (THA) is frequently performed in patients with end-stage hip disease, such as primary osteoarthritis, osteonecrosis, and developmental hip dysplasia of the hip, and is highly successful (1). THA can relieve pain, enhance function, and improve the quality of life of patients (2). Accordingly, THA has become one of the most common surgical procedures in orthopedics and is expected to be increasingly performed with the annual aging of the population $(3,4)$. 
Osteophytes around the acetabulum are common during THA $(5,6)$. However, they should be removed appropriately to avoid potential impingement between osteophytes and femoral prostheses and decrease dislocation risk (7-10). Nevertheless, there are no current standard procedures or surgical technique criteria to remove osteophytes. Surgeons commonly use an osteotome or rongeurs to remove protruding osteophytes. However, such usual practices present some disadvantages. First, there are no clear boundaries between osteophytes and the real acetabular edge, meaning that surgeons may always be in a dilemma regarding the resection range and extent. Traditionally, surgeons may have to repeat the resection procedure several times to achieve satisfactory outcomes, with a high possibility of either excessive removal or incomplete removal. Moreover, the acetabular edge is usually roughened by the aforementioned resection procedure and needs to be repeatedly trimmed. The abovementioned procedures will inevitably prolong the operating time, and excessive osteophyte removal may increase intraoperative acetabular fracture and bleeding risks. Moreover, although rare, there is an additional risk of injury to neurovascular structures (11). Accordingly, the traditional acetabular osteophyte resection procedure is inefficient for proper removal, is unprecise, and has safety concerns. Therefore, this study introduced a novel surgical device and resection procedure, the SH-9Hospital acetabular edge file, which can achieve high efficiency and precisely remove acetabular osteophytes during THA. Thus, this study aimed to introduce the use of the SH-9Hospital acetabular edge file for removing acetabular osteophytes in clinical practice and evaluate the efficiency and precision of this novel surgical device and technique.

We present the following article in accordance with the STROBE reporting checklist (available at https://dx.doi. org/10.21037/atm-21-2400).

\section{Methods}

The study was conducted in accordance with the Declaration of Helsinki (as revised in 2013). The study was approved by Ethics Committee of Shanghai Ninth People's Hospital affiliated to Shanghai Jiao Tong University School of Medicine (approval number: SH9H-2021-T121-2) and individual consent for this retrospective analysis was waived.

\section{Patients}

We retrospectively followed-up 28 patients who underwent
THA with the SH-9Hospital acetabular edge file and 26 patients who underwent THA with an osteotome between January, 2020, and January, 2021. The inclusion criteria were patients eligible for primary THA due to hip diseases and a corresponding computed tomography (CT) scanning that identified osteophytes in need of removal intraoperatively. The exclusion criterion was patients whose data were insufficient. Patients having periacetabular osteophytes were identified using radiography and CT scans preoperatively. In the osteotome group, all 26 patients underwent acetabular osteophyte removal using an osteotome ( 9 male and 17 female, $66.64 \pm 6.29$ y), while in the acetabular edge file group, all 28 patients underwent acetabular osteophyte removal using the SH-9Hospital

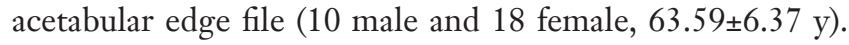
Patient demographic data are presented in Table 1.

\section{SH-9Hospital acetabular edge file}

The SH-9Hospital acetabular edge file is shown in Figure 1. This acetabular edge file was composed of two components with various sizes-the edge file (Figure 1A) and the hemispherical guider (Figure 1B) — which could be assembled together (Figure 1C). The hemispherical guider was placed in the acetabulum in the desired abduction and anteversion orientations after the acetabulum was successfully reamed (Figure 2A,2B). Surgeons were required to choose one of the various diameters of the hemispherical guider to match the acetabulum diameter of each patient. The center of the hemispherical guider comprised a cylindrical connector, which connected with and acted as a guide for the edge file component. The edge file component comprised a disc-shaped structure whose edge was serrated. Surgeons were able to choose different serration widths according to the size of periacetabular osteophytes. The file component had a joint lever that connected with the driving device such as an electric drill. Surgeons were able to remove periacetabular osteophytes precisely and efficiently while the file component rotated at high speeds along the guider-leaded plane (Figure 2C,2D).

\section{Surgical technique}

All operations were performed by the same senior surgeon with rich experience (annual surgical volume of THA was 300-500) using a posterolateral approach under general anesthesia. After dislocating the femoral head and performing a femoral neck osteotomy, osteophytes at the 
Table 1 Patient demographic data in the osteotome and acetabular edge file groups

\begin{tabular}{|c|c|c|c|}
\hline Variables & $\begin{array}{l}\text { Osteotome } \\
\text { group }(\mathrm{N}=26)\end{array}$ & $\begin{array}{l}\text { Acetabular edge file } \\
\text { group }(\mathrm{N}=28)\end{array}$ & $P$ value \\
\hline $\begin{array}{l}\text { Age (years), } \\
\text { mean (SD) }\end{array}$ & $66.64(6.29)$ & $63.59(6.37)$ & 0.083 \\
\hline Gender & & & 0.933 \\
\hline Male & 9 & 10 & \\
\hline Female & 17 & 18 & \\
\hline $\mathrm{BMI}\left(\mathrm{kg} / \mathrm{m}^{2}\right)$ & & & 0.238 \\
\hline$>25$ & 18 & 15 & \\
\hline$\leq 25$ & 8 & 13 & \\
\hline Operation side & & & 0.276 \\
\hline Right & 15 & 12 & \\
\hline Left & 11 & 16 & \\
\hline $\begin{array}{l}\text { Etiology of } \\
\text { indications }\end{array}$ & & & 0.972 \\
\hline OA & 5 & 6 & \\
\hline ONFH & 9 & 9 & \\
\hline $\mathrm{DDH}$ & 12 & 13 & \\
\hline
\end{tabular}

Data are shown as numbers or means (standard deviation). Continuous variables used independent sample $t$-test, and categorical variables used chi-squared test to compare the two groups. N, number; SD, standard deviation; BMI, body mass index; OA, osteoarthritis; ONFH, osteonecrosis of femoral head; $\mathrm{DDH}$, developmental dysplasia of the hip.

bottom and inferomedial area and around the acetabulum circumference were carefully evaluated. A small acetabular reamer was used to vertically ream osteophytes at the bottom and inferomedial area of the acetabulum until the cotyloid fossa was exposed. Subsequently, the acetabulum was reamed until a satisfactory size and coverage were achieved. Before milling the acetabular edge osteophytes, three or four Hohmann retractor were placed around the acetabulum, which was general operating process in total hip arthroplasty surgery. Through this way, soft tissues including nerves and vessels could be separated and protected. Thereafter, osteophytes around the acetabulum were removed using either an osteotome or acetabular edge file. In the SH-9Hospital acetabular edge file group (Figures 3,4 and Video S1), surgeons placed the guider, which had the same size as the final acetabuloplastic reamer, in the acetabulum with the desired orientation. Subsequently, surgeons placed the edge file along the guider and filed acetabular edge osteophytes until the edge file contacted the guider. Since the hemispheric guider had the same size as the cup component and was placed in a specific desired orientation, the filed acetabular edge plane was in high accordance with the implanted cup rim. Meanwhile, in the osteotome group, the surgeon routinely removed acetabular osteophytes according to his experience and judgment. After removing osteophytes, surgeons conducted the same procedures in both groups until a definitive prosthesis was assembled.

\section{Clinical and radiographic evaluations}

Clinical and radiographic data were obtained for all patients intra- and postoperatively. The intraoperative evaluation included the periacetabular osteophytes removal time, which was calculated as the time between the beginning and final periacetabular osteophytes removal. The Harris hip score (HHS) and Visual Analogue Score (VAS) were used to assess hip joint function preoperatively and 3 months postoperatively. Radiography and CT scans were obtained pre- and postoperatively to assess acetabular osteophytes.

\section{Statistical analysis}

SPSS software (Version 19; SPSS Inc., Chicago, IL, USA) was used for statistical analysis. Student's $t$-test was used to compare the mean values of clinical results. A $\mathrm{P}$ value $<0.05$ was considered statistically significant.

\section{Results}

Intraoperative images are shown in Figure 4. Acetabular osteophytes were removed efficiently and precisely in the acetabular edge file group. There were no intraoperative iatrogenic neurovascular injuries or fractures in both groups. The mean HHS and VAS pre- and postoperatively in both groups are shown in Table 2. Both groups significantly improved and had pain relief three months postoperatively. Intraoperatively, the edge file group showed less bleeding than that in the osteotome group; however, this difference was insignificant.

It should be indicated that regarding the osteophyte removal time, the acetabular osteophyte removal time in the osteotome and acetabular edge file groups was $274.6 \pm 102.7$ and $51.3 \pm 21.1 \mathrm{~s}(\mathrm{P}<0.05)$, respectively, indicating that the edge file used significantly less time was much more 

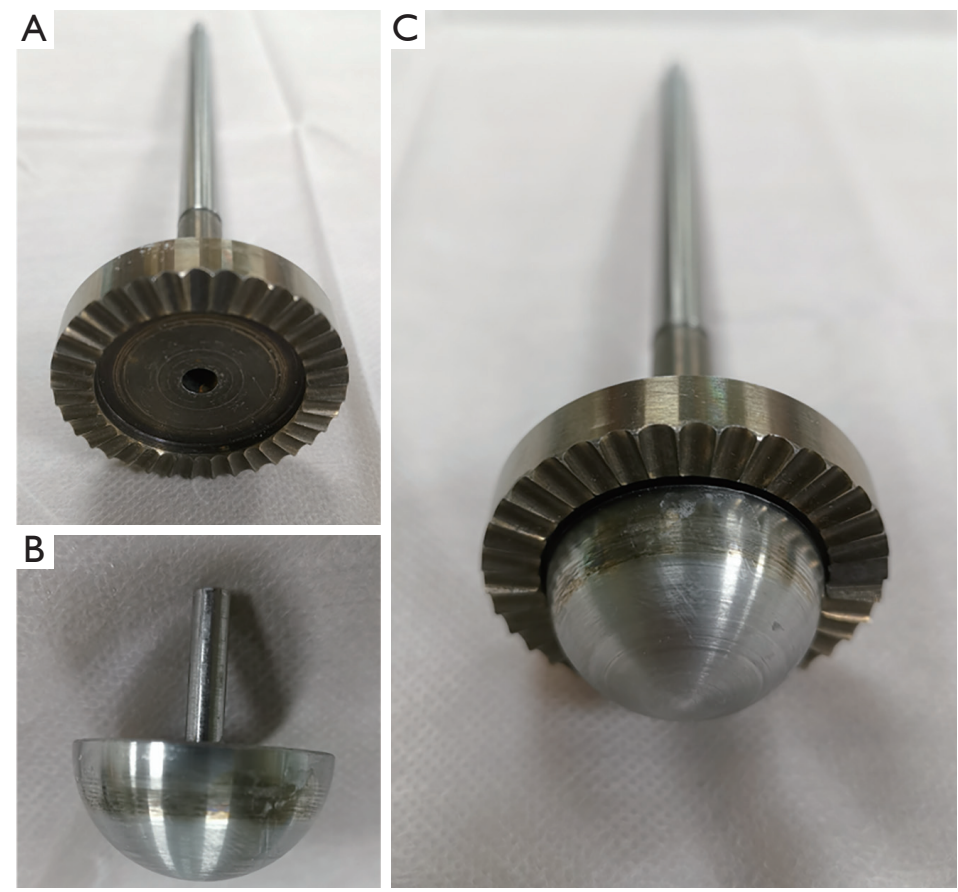

Figure 1 Components of the SH-9Hospital acetabular edge file. (A) Edge file, (B) guider, and (C) assembly.
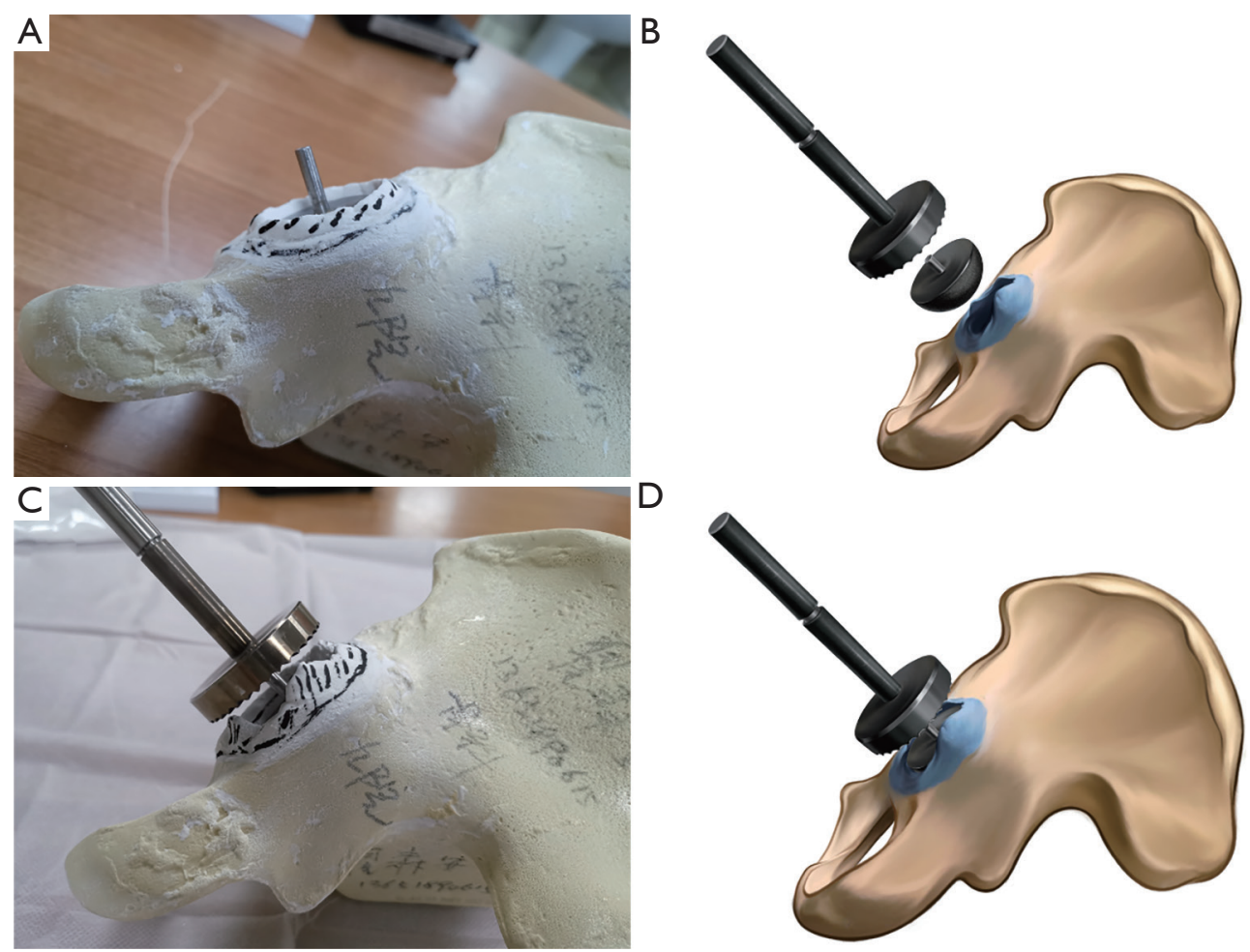

Figure 2 The model and schematic diagram of the removal of acetabular osteophytes using the acetabular edge file. (A,B) Acetabular osteophyte model. Placing the hemispherical guider in the acetabulum in a desired abduction and anteversion orientation. (C,D) Periacetabular osteophytes were filed along the guider-leaded plane and orientation. 


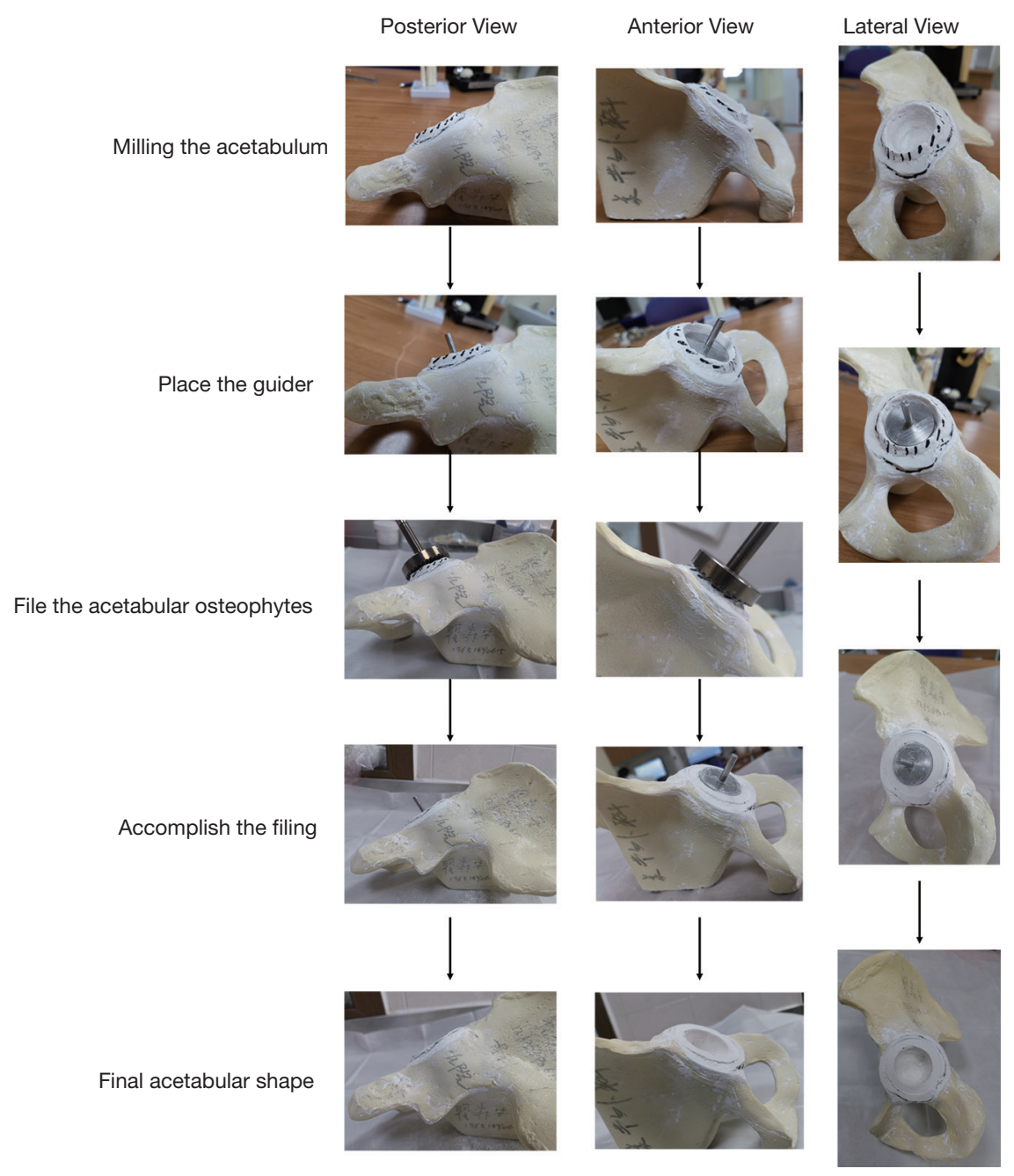

Figure 3 The application procedure of the SH-9Hospital acetabular edge file. The acetabulum was filed, the guider was placed, the osteophytes were filed, and the final acetabular shape was obtained.

efficient than that in the osteotome group.

Preoperative and postoperative radiographs and CT scans showed that acetabular osteophytes were removed thoroughly and precisely by the acetabular edge file (Figures S1,S2). No prosthesis malposition or dislocation was observed in either group.

\section{Discussion}

THA is currently an effective method to treat end-stage hip disease $(12,13)$; however, osteophytes are common in this procedure $(5,6)$. Impingement between acetabular osteophytes and femoral prostheses can cause various problems, including limited motion, subluxation, dislocation, and acetabular liner damage $(7-10,14,15)$. It is suggested that acetabular osteophytes should be removed intraoperatively to avoid potential impingement (7-10). However, a precise and efficient method for removing osteophytes is not available thus far. Insufficient osteophyte removal may lead to residual impingement, while excessive osteophyte removal may lead to bone stock loss and even further increase acetabular fracture or bleeding risk. Such osteophytes are commonly removed using an osteotome. However, this method is ineffective, inefficient, and highly relies on the personal experience and judgment of surgeons. Moreover, obtaining a regular surface paralleled with the acetabular cup rim is challenging, and an osteotome may increase intraoperative acetabular fracture or cup loose risk. In this study, we evaluated a novel and efficient 

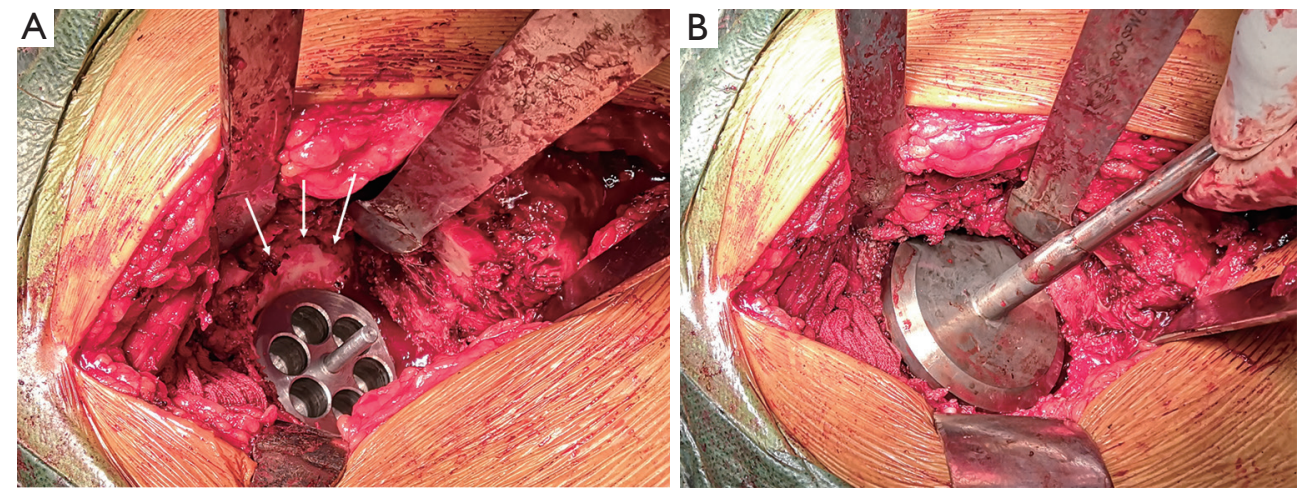

Figure 4 Intraoperative photographs of the application of the SH-9Hospital acetabular edge file. (A) Placing the hemispherical guider in the acetabulum in a desired abduction and anteversion orientation. White arrows show acetabular osteophytes. (B) Periacetabular osteophytes were filed along the guider-leaded plane and orientation.

Table 2 Comparison of clinical results between the osteotome and acetabular edge file groups

\begin{tabular}{lcc}
\hline Index & Osteotome group $(\mathrm{M} \pm \mathrm{SD})$ & Acetabular edge file group $(\mathrm{M} \pm \mathrm{SD})$ \\
\hline Preoperative HHS & $41.4 \pm 6.8$ & $44.8 \pm 8.2$ \\
Postoperative HHS & $86.6 \pm 8.3$ & $89.3 \pm 7.2$ \\
P value & $\mathrm{P}<0.05$ & $\mathrm{P}<0.05$ \\
Preoperative VAS & $7.5 \pm 1.9$ & $7.9 \pm 1.8$ \\
Postoperative VAS & $2.6 \pm 1.2$ & $2.3 \pm 1.1$ \\
P value & $\mathrm{P}<0.05$ & $\mathrm{P}<0.05$ \\
Blood loss $(\mathrm{mL})$ & $263.8 \pm 63.9$ & $237.9 \pm 89.6$ \\
Osteophyte removing time $(\mathrm{s})$ & $274.6 \pm 102.7$ & $51.3 \pm 21.1$ \\
\hline
\end{tabular}

Data are shown as numbers or means (standard deviation). M, mean; SD, standard deviation; HHS, Harris hip score; VAS, Visual analogue score.

surgical technique, the acetabular edge file, that achieved satisfactory and definite outcomes.

In this method, surgeons performed acetabular reaming first and subsequently placed the guider, which had the same diameter as the reamer, in the desired orientation. Accordingly, the guider edge was equal to the implanted cup edge, and after osteophytes above the guider edge were filed, surgeons were able to precisely obtain the acetabular edge, which was further verified by post-operative CT and radiography images. The osteophyte could be resected reliably, there was no insufficient or excessive resection using the edge file. Although there were potential of acetabular fractures, none intraoperative iatrogenic neurovascular injuries or fractures were found in the edge file group. Osteophyte removal using the SH-9Hospital acetabular edge file was highly precise, reliably and safely. It is worthy to note that the clinical data in this study also supported the application of the SH-9Hospital acetabular edge file. In the edge file group, patients obtained significant functional improvement and pain relief, blood loss did not increase, and more importantly, the osteophyte removal time was significantly reduced compared to that in the osteotome group. Although approximately only 3 min were saved in the edge file group compared to that in the osteotome group, we believe that this time is important. A prolonged operational time may increase surgical complication risk (16). Operation time is an important factor related to intraoperative security and the postoperative rehabilitation of patients, especially when most patients undergoing THA are old $(17,18)$. Thus, a reduced osteophyte removal time in the acetabular edge file group can decrease anesthetic time and, possibly, anaesthesia-associated complications in 
patients, and potentially accelerate the operative process.

Briefly, the SH-9Hospital acetabular edge file (I) significantly reduced osteophyte removal time and was highly efficient. (II) This technique also filed the acetabular edge along the guider in a desired orientation, making the acetabular edge equal to implanted cup anteversion and abduction angles parallel to the cup rim, and was highly precise. (III) It also provided the surgeons a standardized operation procedure, reduced the previous high reliability on the personal experience and judgement of surgeons, and achieved high sustainability and repeatability. Accordingly, the SH-9Hospital acetabular edge file is a promising surgical tool and technique with a high potential to be applied widely in THA surgeries in the future.

Our study has several limitations. First, the sample size of the study group was relatively small, meaning that more clinical acetabular edge file operations are needed in the future to assess whether an acetabular edge file can reduce the acetabular bone fracture ratio while removing osteophytes. Moreover, more long-term follow-ups are needed to assess hip function, the dislocation ratio, and acetabular cup survival rate. Third, it is important to note that using the SH-9Hospital acetabular edge file may lead to thorough soft tissue exposure around the acetabulum.

\section{Conclusions}

We presented a novel and efficient surgical technique, the SH-9Hospital acetabular edge file, for removing periacetabular osteophytes. Osteophyte removal using the SH-9Hospital acetabular edge file was highly efficient, precise, repeatable, easy to perform, and achieved an acetabular bone surface parallel to the cup rim without intraoperative iatrogenic injury.

\section{Acknowledgments}

Funding: This study was supported by the Shanghai Municipal Key Clinical Specialty (shslczdzk00402, shslczdzk07001); The Youth Doctor Collaborative Innovation Team Project of Shanghai Ninth People's Hospital, Shanghai Jiaotong University School of Medicine (QC201903).

\section{Footnote}

Reporting Checklist: The authors have completed the STROBE reporting checklist. Available at https://dx.doi. org/10.21037/atm-21-2400

Data Sharing Statement: Available at https://dx.doi. org/10.21037/atm-21-2400

Peer Review File: Available at https://dx.doi.org/10.21037/ atm-21-2400

Conflicts of Interest: All authors have completed the ICMJE uniform disclosure form (available at https://dx.doi. org/10.21037/atm-21-2400). The authors have no conflicts of interest to declare.

Ethical Statement: The authors are accountable for all aspects of the work in ensuring that questions related to the accuracy or integrity of any part of the work are appropriately investigated and resolved. The study was conducted in accordance with the Declaration of Helsinki (as revised in 2013). The study was approved by Ethics Committee of Shanghai Ninth People's Hospital affiliated to Shanghai Jiao Tong University School of Medicine (approval number: SH9H-2021-T121-2) and individual consent for this retrospective analysis was waived.

Open Access Statement: This is an Open Access article distributed in accordance with the Creative Commons Attribution-NonCommercial-NoDerivs 4.0 International License (CC BY-NC-ND 4.0), which permits the noncommercial replication and distribution of the article with the strict proviso that no changes or edits are made and the original work is properly cited (including links to both the formal publication through the relevant DOI and the license). See: https://creativecommons.org/licenses/by-nc-nd/4.0/.

\section{References}

1. Ferguson RJ, Palmer AJ, Taylor A, et al. Hip replacement. Lancet 2018;392:1662-71.

2. Higgins BT, Barlow DR, Heagerty NE, et al. Anterior vs. posterior approach for total hip arthroplasty, a systematic review and meta-analysis. J Arthroplasty 2015;30:419-34.

3. Mellon SJ, Liddle AD, Pandit H. Hip replacement: landmark surgery in modern medical history. Maturitas 2013;75:221-6.

4. Learmonth ID, Young C, Rorabeck C. The operation of the century: total hip replacement. Lancet 2007;370:1508-19.

5. Mao Y, Yu D, Xu C, et al. The fate of osteophytes in the 
superolateral region of the acetabulum after total hip arthroplasty. J Arthroplasty 2014;29:2262-6.

6. Nevalainen MT, Kauppinen KV, Niinimki T, et al. Comparison of ultrasonographic, radiographic and intraoperative findings in severe hip osteoarthritis. Sci Rep 2020;10:21108.

7. Rodriguez-Elizalde S, Yeager AM, Ravi B, et al. Computerized virtual surgery demonstrates where acetabular rim osteophytes most reduce range of motion following total hip arthroplasty. HSS J 2013;9:223-8.

8. Kurtz WB, Ecker TM, Reichmann WM, et al. Factors Affecting Bony Impingement in Hip Arthroplasty ScienceDirect. J Arthroplasty 2010;25:624-34.e2.

9. Shoji T, Yamasaki T, Izumi S, et al. Factors affecting the potential for posterior bony impingement after total hip arthroplasty. Bone Joint J 2017;99-B:1140-6.

10. Shoji T, Ota Y, Saka H, et al. Factors affecting impingement and dislocation after total hip arthroplasty - Computer simulation analysis. Clin Biomech (Bristol, Avon) 2020;80:105151.

11. Kim JT, Lee J, Lee YK, et al. What is the tolerated width of periacetabular osteophytes to avoid impingement in cementless THA?: a three-dimensional simulation study.

Cite this article as: Chang Y, Kong K, Zeng Y, Yu D, Zhang J, Yan M, Mao Y, Li H, Zhai Z. A novel efficient and precise technique for removing acetabular osteophytes in patients undergoing total hip arthroplasty: the SH-9Hospital acetabular edge file. Ann Transl Med 2021;9(17):1366. doi: 10.21037/atm21-2400
Arch Orthop Trauma Surg 2018;138:1165-72.

12. Evans JT, Walker RW, Evans JP, et al. How long does a knee replacement last? A systematic review and metaanalysis of case series and national registry reports with more than 15 years of follow-up. Lancet 2019;393:655-63.

13. Pivec R, Johnson AJ, Mears SC, et al. Hip arthroplasty. Lancet 2012;380:1768-77.

14. Malik A, Maheshwari A, Dorr LD. Impingement with total hip replacement. J Bone Joint Surg Am 2007;89:1832-42.

15. Dargel J, Oppermann J, Bruggemann GP, et al. Dislocation following total hip replacement. Dtsch Arztebl Int 2014;111:884-90.

16. Peersman G, Laskin R, Davis J, et al. Prolonged operative time correlates with increased infection rate after total knee arthroplasty. HSS J 2006;2:70-2.

17. Gowd AK, Liu JN, Bohl DD, et al. Operative Time as an Independent and Modifiable Risk Factor for ShortTerm Complications After Knee Arthroscopy. Arthroscopy 2019;35:2089-98.

18. Bohl DD, Ondeck NT, Darrith B, et al. Impact of Operative Time on Adverse Events Following Primary Total Joint Arthroplasty. J Arthroplasty 2018;33:2256-62.e4. 
Case 1
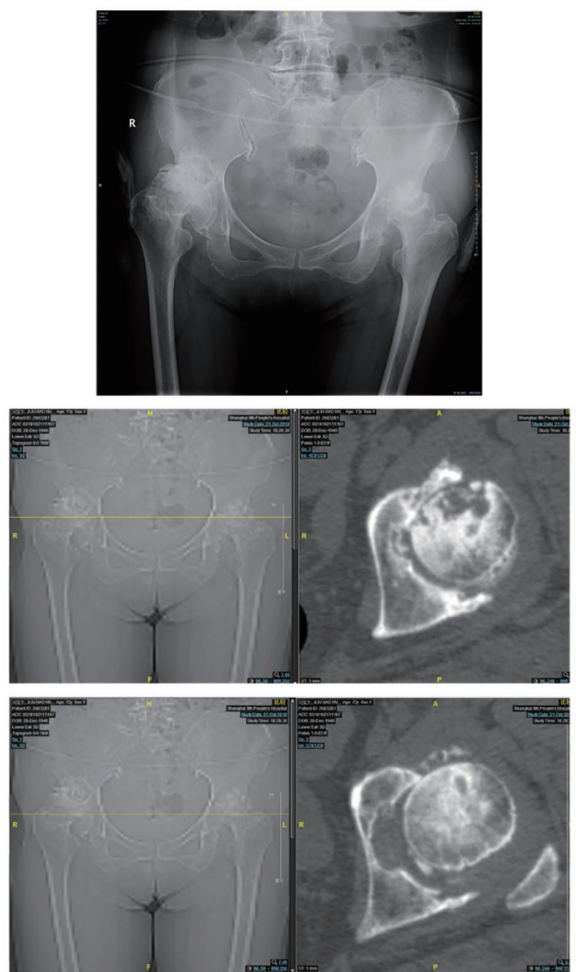

Postoperation
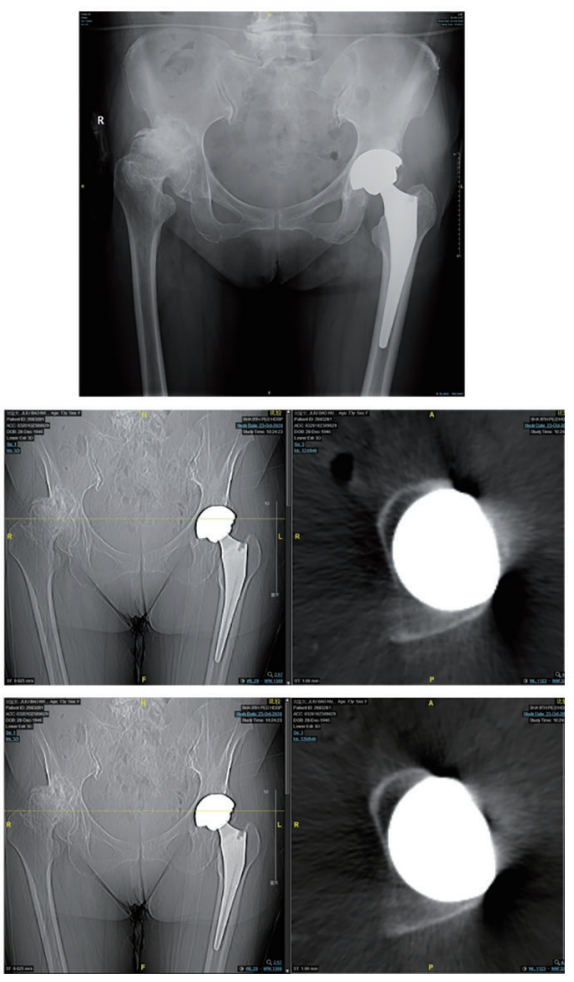

Figure S1 Radiographs of patient 1 in the acetabular edge file group postoperatively.

Case 2
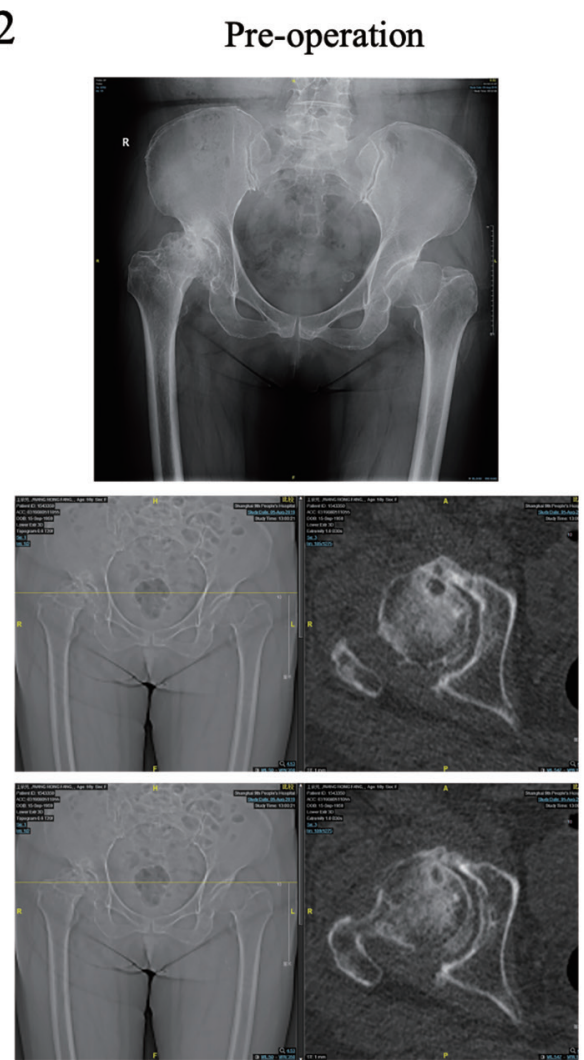

Postoperation
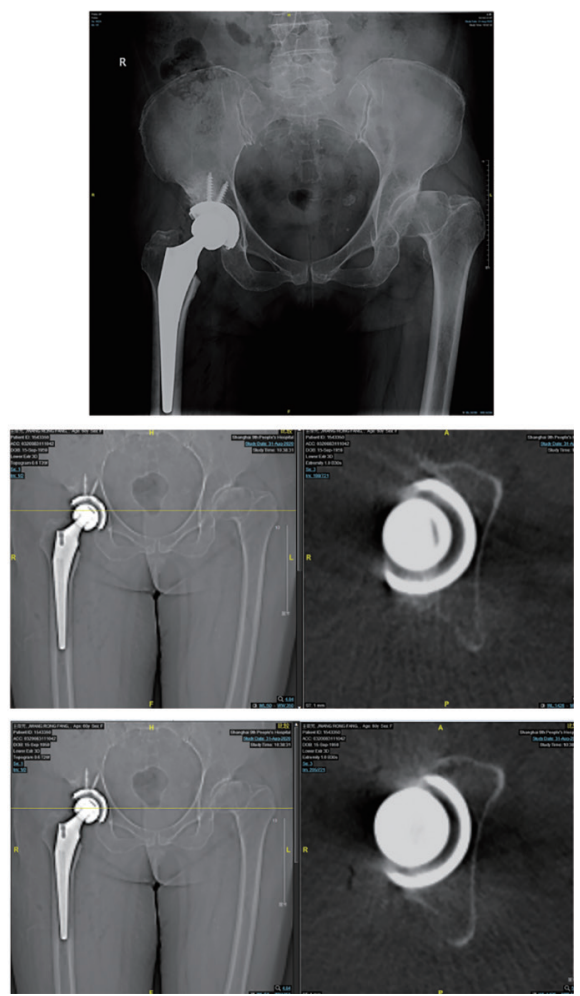

Figure S2 Radiographs of patient 2 in the acetabular edge file group postoperatively. 